\title{
Vom Sichtbarmachen des Unsichtbarwerdens - vom Unsichtbarmachen des Sichtbarwerdens
}

Pierre Bühler

Dass Geschichten,in denen von den Erscheinungen des Auferstandenen erzählt wird, mit visuellen Aspekten arbeiten, mag auf der Hand liegen. Überraschendes Sichtbar- und Unsichtbarwerden gehört wohl wesenhaft dazu. Trotzdem fällt in dieser Hinsicht eine Erscheinungsgeschichte ganz besonders auf, nämlich die Erzählung von der Erscheinung des auferstandenen Christus auf dem Weg nach Emmaus in Lukas 24.

\section{Die visuelle Spannung von Sichtbar- und Unsichtbarwerden in Lukas 24}

Jesus gesellt sich zu zwei Jüngern, die auf dem Weg von Jerusalem $\mathrm{zu}$ einem Dorf namens Emmaus sind. Kaum hat die Begegnung stattgefunden, fällt auch der erste visuelle Kommentar des Erzählers: "Doch ihre Augen waren gehalten, so dass sie ihn nicht erkannten.» (V. 16) ${ }^{1}$ Die gehaltenen Augen sehen ihn also, aber erkennen ihn nicht, oder anders gesagt: Er erscheint den Jüngern sichtbar, aber seine eigentliche Identität bleibt ihnen verborgen, unsichtbar. Im Markusevangelium hatte Jesus diese Spannung auch bereits thematisiert, in Anlehnung an Jesaja 6, 9-10: Denen, die draussen sind, hiess es dort, werde alles in Gleichnissen erzählt, «damit sie sehend sehen und nicht erkennen».

Aufgrund dieses Nichterkennens nimmt nun die Geschichte für den Leser, der ja weiss, wer sich da zu den zwei Jüngern gesellte, eine ironische Wendung. Auf die Frage, was sie denn für Worte miteinander wechselten, antwortet der eine der Jünger dem Auferstandenen: «Du bist wohl der Einzige, der sich in Jerusalem aufhält und nicht erfahren hat, was sich in diesen Tagen dort zugetragen hat.» (V. 18) Der Nichtsahnende vermutet, der Unbekannte habe von all dem, was sich zugetragen hat, nichts erahnt, und dabei war ja gerade dieser Unbekannte im Zentrum dieses Geschehens! Das Missverständnis

\footnotetext{
Ich zitiere die Geschichte nach der in diesem Jahr erschienenen revidierten Fassung der Zürcher Bibel, Zürich, Genossenschaft Verlag der Zürcher Bibel beim TVZ, 2007.
} 
wird nicht aufgehoben, und so kann es denn weiterhin die narrative Spannung steigern: Als ob er von allem nichts wüsste, lässt sich der auferweckte Jesus von den Jüngern die ganze Geschichte seiner Passion und Auferstehung nacherzählen! Interessanterweise schliesst dieser lange Bericht mit dem Hinweis: «ihn aber haben sie nicht gesehen». Auch dies enthält ironisches Potential für den Leser: Die, die ihn weiterhin nicht «sehen», nicht erkennen, erzählen ihm von anderen, die ihn nicht gesehen haben. Die Spannung von Sichtbar- und Unsichtbarwerden wird mit dieser Verschachtelung der Erzählung in der Erzählung narrativ redupliziert.

Um dann bei seinen Jüngern die Unverständigkeit und die Trägheit des Herzens zu bekämpfen, wird nun der Auferweckte zum

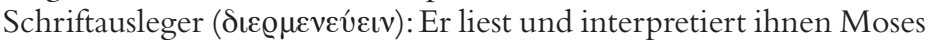
und alle Propheten (V. 27) in Hinsicht darauf, was hier über ihn selbst zu lesen ist. Auch Schriftauslegung ist visuelle Tätigkeit, was etwas später, rückblickend, als das «Aufschliessen» der Schriften zum Ausdruck kommt (V. 32). Hermeneutisch gesehen ist der Kontrast spannend: Währenddem die Augen immer noch verschlossen sind, werden die Schriften «aufgeschlossen», und zwar gerade durch den noch nicht erkannten Auferstandenen!

Der narrative Höhepunkt in der Spannung von sichtbar und unsichtbar geschieht schliesslich nach der Ankunft in Emmaus, und zwar auch hier eindeutig mit einer visuellen Konzentration:Was sich im Laufe des Unterwegsseins an "Öffnungsarbeit» bereits vollziehen konnte, findet in der elementaren Geste des Brotbrechens und -segnens seinen Abschluss, und zwar einen Abschluss in äusserster Paradoxie. In Verbindung mit dieser Geste heisst es: «Da wurden ihnen die Augen aufgetan, und sie erkannten ihn.»(V. 31) Waren in V. 16 die Augen noch gehalten, so dass keine Erkenntnis möglich wird, sind nun dieselben Augen aufgetan und können deshalb erkennen. Doch, und hier geschieht das Paradox: «Und schon war er nicht mehr zu sehen.» Das heisst: Aus einem langwierigen, komplexen Sichtbarwerden entsteht gleich wieder augenblicklich ein unmittelbares Unsichtbarwerden. Den sichtbar Gewordenen hat man nie im Griff, man kann ihn nicht einfangen, nicht über ihn verfügen. Kaum ist er im tiefsten Sinne sichtbar, erkannt, ist er auch schon wieder unsichtbar. Wer hier unversehens erkannt wurde, der wird nun zum Gegenstand einer Verkündigung (V. 33-35), nicht etwa eines kontemplativen Schauens. Seine Begleitung kann nun die eines Wortes werden, das es weiterzutragen gilt, und wohl auch des Sakraments, das sich mit dem Brotbrechen und -segnen verbinden wird, des 
Abendmahls, in dem eine sichtbar-unsichtbare Realpräsenz Christi erfahren werden kann.

So bemüht sich dieser Text durch und durch, das Sichtbarwerden unsichtbar zu machen und das Unsichtbarwerden sichtbar zu machen, durch die narrative Umsetzung einer schöpferischen Hermeneutik des Missverständnisses. Könnte das, was hier erzählerisch geschieht, auch bildnerisch zum Vollzug kommen?

\section{Bilder des Unsichtbarwerdens?}

«Die Kirche, die einmal die Bilder instrumentalisierte, müsste eigentlich heute, wenn ich einmal so utopisch reden darf, die Bildlosigkeit, die Transzendenz des Bildes, sie müsste die Unsichtbarkeit und die Undarstellbarkeit der grossen Themen des Menschen, der Religion schützen und propagieren. Sie müsste Bildaskese betreiben. Sie müsste wieder ins Bewusstsein bringen, dass ebenso wenig, wie sich die Welt in Informationen begreifen lässt, sich die Welt und die Dinge, die uns wirklich wichtig sind, darstellen lassen. Was uns wirklich wichtig ist, davon lässt sich kein Bild machen.» ${ }^{2}$

Die Instrumentalisierung, die Hans Belting der Kirche vorwirft, hat mit einem unmittelbaren Sichtbarmachen zu tun, in dem das Dargestellte greifbar, verfügbar, und deshalb auch religiös brauchbar, umsetzbar wird.Demgegenüber betont er seine (utopische) Bildaskese in einem radikalen Sinne:Verzicht darauf, das wirklich Wichtige im Bild darzustellen, oder die Bildlosigkeit, die Unsichtbarkeit und die Undarstellbarkeit in Hinsicht auf die grossen Themen des Menschen, der Religion zu propagieren.

Es muss hier kritisch gefragt werden: Besteht damit nicht die Gefahr, in eine falsche Alternative zu geraten? Von einer instrumentalisierenden, manipulierbaren Sichtbarkeit in eine strenge, eben asketische, sich isolierende Unsichtbarkeit, die dann auch eine Suspension der Darstellung überhaupt, eine Aufhebung der Visualität impliziert? Demgegenüber wäre hervorzuheben, dass Visualität in einem dynamischen Sinne vielmehr im Grenzbereich zwischen dem Sichtbaren und dem Unsichtbaren angesiedelt ist und es stets mit den Übergängen vom einen in das andere zu tun hat. Instrumentalisierende Sichtbarkeit ist eine von der Unsichtbarkeit

2 H. Belting, Skizzen zur Bilderfrage und zur Bilderpolitik heute, in: E. Nordhofen (Hg.), Bilderverbot. Die Sichtbarkeit des Unsichtbaren, Paderborn, Schöning, 2001, 36; zitiert nach: M. Krieg u. a. (Hg.), Das unsichtbare Bild. Die Ästhetik des Bilderverbotes, Zürich, TVZ, 2005, 83. 
losgelöste Sichtbarkeit, und Undarstellbarkeit wäre der radikale Verzicht auf Sichtbarmachen, weil dieses erstarrend, einfangend, vereinnahmend wirkt.

Um diese Gegenüberstellung ein Stück weit zu überwinden, zu dynamisieren, könnte man sich überlegen, ob Bildaskese nicht gerade an dieser Grenze wacht, an den Grenzübergängen arbeitet, damit sie das unverfügbar Unsichtbare greifbar sichtbar werden lassen, um zugleich das greifbar Sichtbare erneut in unverfügbar Unsichtbares $\mathrm{zu}$ verwandeln. Also nicht: Sichtbarkeit vs. Unsichtbarkeit, sondern dynamische Visualität im Spannungsfeld von Sichtbarkeit und Unsichtbarkeit. Erst dann ist so etwas wie «die Transzendenz des Bildes» im Vollzug.

Das ist genau das Anliegen des erzählerischen Sichtbarmachens des Unsichtbaren sowie des Unsichtbarmachens des Sichtbaren in Lukas 24. Im christlichen Glauben gehört die frohe Botschaft, dass der Auferstandene mit den Gläubigen unterwegs ist, zum wirklich Wichtigen, zu den grossen Themen des Menschen, der Religion, im Sinne von Belting. Dieses «Unterwegssein mit ...» muss zugleich greifbar sichtbar und unverfügbar unsichtbar sein. Nur so wird man diesem "wirklich Wichtigen» gerecht.

Liesse sich das auch im Bild vollziehen?

\section{«Christus in Emmaus» bei Rembrandt}

Um an der formulierten Frage zu arbeiten, möchte ich zwei Bilder von Rembrandt heranziehen, die meiner Ansicht nach bildnerisch genau an dieser Spannung arbeiten. Beide tragen den Titel «Christus in Emmaus», und nicht von ungefähr setzen beide auch an der Situation an, die wir als narrativen Höhepunkt von Lukas 24 bezeichnet haben, nämlich am Tisch im Haus, beim Brotbrechen und -segnen.

Im ersten Bild, von 1629, fällt zunächst einmal auf, dass nur noch einer der zwei Jünger mit Jesus am Tisch sitzt, währenddessen der andere im dunklen Vordergrund des Bildes vom Stuhl gefallen ist, sehr wahrscheinlich vor Entsetzen. Auf jeden Fall lässt sich am staunenden, ja sogar entsetzten Blick des noch sitzenden Jüngers ablesen, dass ein Erkennungsvorgang stattfindet, der für den anderen Jünger wortwörtlich umwerfend gewirkt hat ${ }^{3}$. Diesen Jüngern wurden

\footnotetext{
3 Der Vordergrund des Bildes ist so dunkel, dass man bei einer schlechten Qualität der Reproduktion den am Boden kriechenden (oder bereits kniend anbetenden?) Jünger nicht sieht. Man könnte dann versucht sein, ihn mit der Gestalt im Hinter-
} 


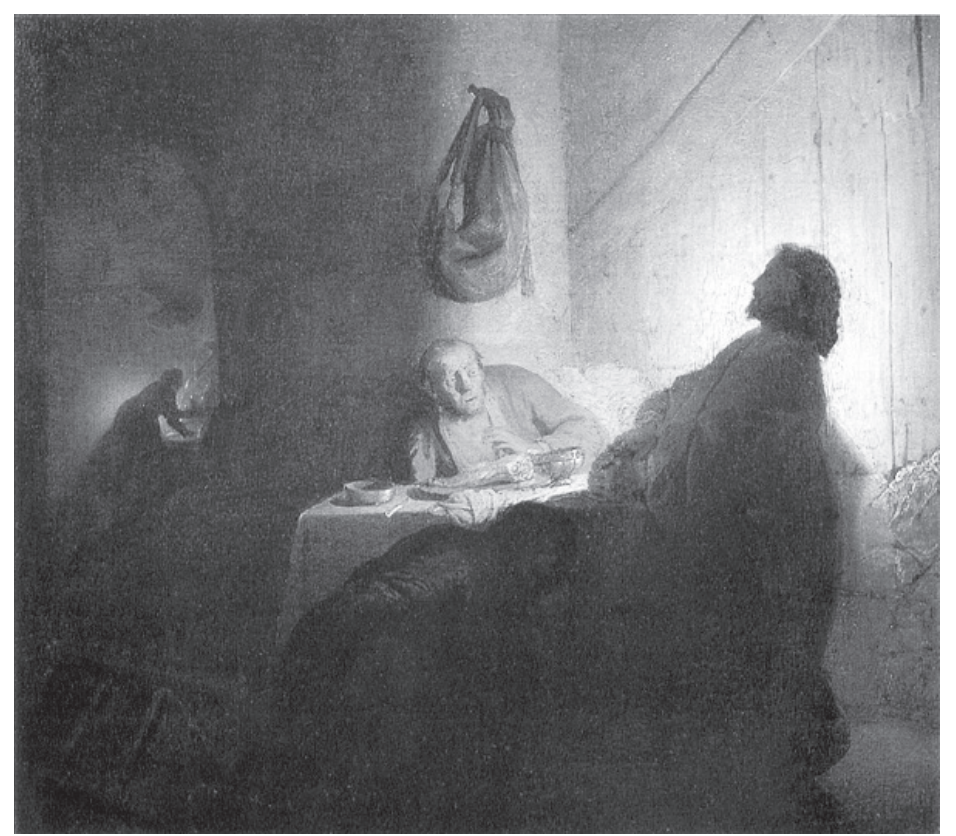

Rembrandt van Rijn, Christus in Emmaus, 1629, Öl auf Papier, auf Holz aufgezogen, Musée Jacquemart-André, Paris.

die Augen aufgetan. Doch heisst das, dass nun der brotbrechende Auferstandene gleich verschwinden wird, wie wir aus der Erzählung wissen. Bildnerisch wird dieses Unsichtbarwerden dadurch sichtbar gemacht, dass der Auferstandene im scharfen Licht-SchattenKontrast bereits zu einer sehr schemenhaften Profilfigur geworden ist. Schon fast sieht man von ihm nur noch einen ungreifbaren Schatten, während er selbst bereits weg ist! Und doch ist interessanterweise das Licht-Schatten-Verhältnis so gestaltet, dass diese schemenhafte, dunkle Figur anscheinend zugleich die Lichtquelle ist. Auf jeden Fall wird der noch sitzende Jünger genau aus dieser Richtung beleuchtet (erleuchtet?). Das Bild ist an der Grenze des Sichtbaren und des Unsichtbaren: Noch sieht der Jünger seinen Gast, noch sieht der Zuschauer die Hände, die soeben das Brot gebrochen haben, und doch wirkt der Auferstandene wie bereits schwindend, nur noch als ein Schatten im Licht wahrnehmbar. «Da wurden ihnen die Augen

grund zu identifizieren; diese ist jedoch eher als eine Dienerin zu interpretieren, die in der Küche arbeitet. 


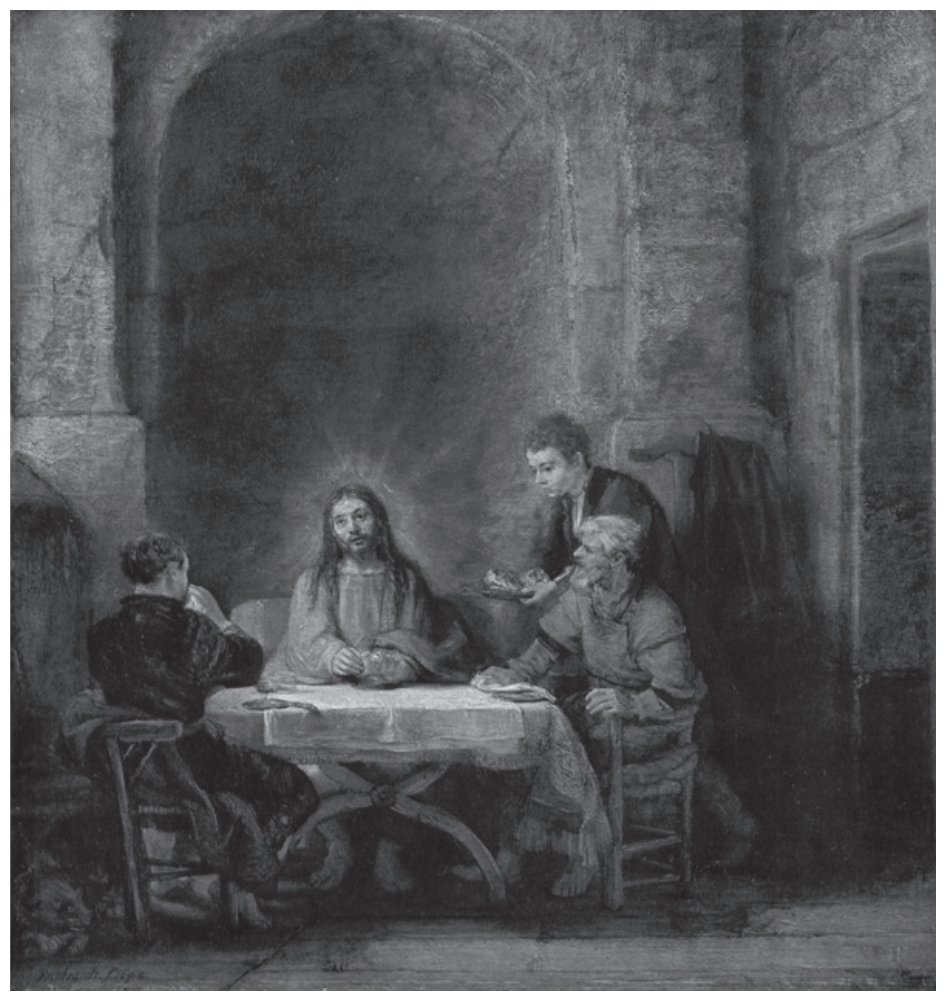

Rembrandt van Rijn, Christus in Emmaus, 1648, Öl auf Holz, Musée du Louvre, Paris.

aufgetan, und sie erkannten ihn. Und schon war er nicht mehr zu sehen.»

Im zweiten Bild, von $1648^{4}$, stehen wir an derselben Grenze, nun aber bildnerisch anders inszeniert. Hier sitzen beide Jünger am Tisch, und zusätzlich steht auf der rechten Seite ein Diener, der die Tischgenossen bedient. Hier sehen wir den auferweckten Jesus nicht im Profil, sondern von vorne. Die hellste Stelle im Bild ist das Tischtuch, ganz in der Nähe der brotbrechenden Hände, während zugleich eine leichte Lichtaura den Kopf des Auferstandenen um-

4 Zu diesem Bild, vgl. auch: J. Stückelberger, Das unsichtbare Bild. Prolegomena zu einer reformierten Ästhetik, in: Krieg (Hg.), Das unsichtbare Bild, s. Anm. 2, 11-19, vor allem $17 \mathrm{f}$. 
gibt. Jesus in Frontalansicht ist hier greifbar nahe, und die Jünger scheinen auch (noch) nicht zu erschrecken. Demgegenüber fällt auf, dass in der oberen Bildhälfte (und es macht wirklich eine ganze Hälfte des Gemäldes aus!) ein grosser Leerraum dargestellt wird, allein aus nackten Mauern bestehend, mit einer grossen dunklen Nische. Der nach oben gerichtete Blick des brotbrechenden und -segnenden Christus verweist den Zuschauer auf diesen hohen leeren Raum. In diesen hinein wird der Auferstandene wohl gleich entschwinden, währenddessen die Augen der sitzenden Jünger geöffnet werden, aber dann auch schon wieder nichts mehr sehen! Die dunkle obere Hälfte des Bildes macht das augenblicklich bevorstehende Unsichtbarwerden Jesu sichtbar: Der Auferstandene kann nur als unverfügbar Unsichtbarer mit seinen Jüngern greifbar unterwegs sein, das Brot brechen.

Und so könnte der Zuschauer im nachhinein in die Frage der Jünger einstimmen: «Brannte nicht unser Herz, als er unterwegs mit uns redete, als er uns die Schriften aufschloss?» (V.32) Ein Stück weit tragen Rembrandts Bilder für den Zuschauer seiner Bilder, hermeneutisch gesehen, zu diesem Aufschliessen der Schriften bei: Das bildnerische Ringen mit dem Sichtbarmachen des Unsichtbarwerdens und dem Unsichtbarmachen des Sichtbarwerdens weist auf dasselbe Ringen in der biblischen Erzählung. Und vielleicht weist es zugleich auf ein anderes, ähnliches Ringen, das sich im Leben des Zuschauers und Lesers redupliziert. Denn wir sind ja auch unterwegs, und allzu oft mit gehaltenen Augen.

—Dr. Pierre Bühler ist Professor für Systematische Theologie, insbesondere Hermeneutik und Fundamentaltheologie an der Universität Zürich. 